\title{
Efecto de diferentes densidades de cultivo en el comportamiento productivo del langostino blanco (Litopenaeus vannamei) (BOONE, 1931) bajo condiciones de invernadero
}

\author{
Effect of different culture densities on growth performance of white shrimp (Litopenaeus \\ vannamei) (Boone, 1931) under greenhouse conditions
}

\author{
Fernando Galecio R. ${ }^{1}$; Karina Vicente C. ${ }^{2}$
}

\begin{abstract}
Resumen
El presente trabajo se llevó a cabo en las instalaciones de la empresa langostinera Domingo Rodas S.A., ubicada en el departamento de Tumbes, con el objetivo de determinar la densidad de siembra más adecuada del langostino (Litopenaeus vannamei) bajo la modalidad intensiva en invernadero. Se utilizó como material biológico 2240 juveniles de Litopenaeus vannamei con un peso promedio de 3,05 gramos, distribuidos al azar en 16 jaulas experimentales con dimensiones de 1 metro de largo x 1 metro de ancho x 1.70 metros de altura, instaladas en un estanque de 1 ha con un sistema de aireación. El cultivo tuvo una duración de 70 días, donde se evaluaron las siguientes densidades de cultivo: $80 \mathrm{unid} / \mathrm{m}^{2}$ (T-1), $120 \mathrm{unid} / \mathrm{m}^{2}$ (T-2), $160 \mathrm{unid} / \mathrm{m}^{2}$ (T-3) y 200unid $/ \mathrm{m}^{2}$ (T-4), con sus respectivas réplicas. Los controles biométricos realizados fueron cada 2 semanas, así como el seguimiento de los parámetros físicos, químicos y biológicos del agua. Los resultados obtenidos reportaron pesos promedios finales de 15,51 g (T-1); 15,61g (T-2); $14,87$ g (T-3); y 14,33 g (T-4); encontrándose diferencia significativa ( $\mathrm{a}=0,05)$, tasas de crecimiento de $2,48,2,51$, 2,36 y 2,27 g., respectivamente; los valores de biomasa final obtenida fueron de 941,33 g, 1384,99 g, $1772,16 \mathrm{~g} \mathrm{y}$ $2068,71 \mathrm{~g}$, respectivamente, con una diferencia significativa $(\mathrm{a}=0,05)$. Las sobrevivencias promedio para cada uno de los tratamientos fueron de 75,94\%,73,96\%,74,53\%, y 72,25\% que no demostraron diferencia significativa $(\mathrm{a}=0,05)$ y el factor de conversión alimenticia (F.C.A.) promedio fue de 2,47; 2,57, 2,69, y 2,92, respectivamente. Respecto de los parámetros físicos, químicos y biológicos del agua, se encontraron dentro de las recomendaciones para el cultivo.

Se utilizó el diseño experimental de mediciones repetidas a través del Análisis de Varianza y la Prueba de Tukey $(\mathrm{a}=0,05)$ y se concluyó que la densidad con la cual se obtuvo los mejores resultados fue con el tratamiento (T-2) de 120 $\mathrm{unid} / \mathrm{m}^{2}$.
\end{abstract}

Palabras clave: Comportamiento productivo; densidades de cultivo; invernadero; Litopenaeus vannamei.

\begin{abstract}
This research was carried out in the facilities of the shrimp Company Domingo Rodas SA, located in the department of Tumbes, with the objective of determining the most suitable stocking density of shrimp (Litopenaeus vannamei) under greenhouse intensive mode. We used 2,240 juvenile Litopenaeus vannamei as biological material, with an average weight of 3.05 grams, randomly distributed in 16 experimental cages with dimensions of 1 meter long $\mathrm{x} 1$ meter wide $\mathrm{x}$ 1.70 meters high, installed in a pond of 1 ha. with an aeration system. The culture lasted 70 days, where the following culture densities were evaluated: 80 unit.m-2 (T-1), 120 unit.m $\mathrm{m}^{-2}$ (T-2), 160 unit.m ${ }^{-2}$ (T-3) and 200 unit.m ${ }^{-2}$ (T-4), with their respective repetitions. Biometric controls were conducted every 2 weeks, as well as the monitoring of physical, chemical and biological water conditions. The results obtained reported average final weights of $15.51 \mathrm{~g}(\mathrm{~T}-1), 15.61$ $\mathrm{g}(\mathrm{T}-2), 14.87 \mathrm{~g}(\mathrm{~T}-3)$, and $14.33 \mathrm{~g}(\mathrm{~T}-4)$; finding significant difference $(\mathrm{a}=0.05)$, growth rates of 2.48, 2.51, 2.36, 2.27 g. respectively; the final biomass values obtained were $941.33 \mathrm{~g}, 1384.99 \mathrm{~g}, 1772.16 \mathrm{~g}$ and $2068.71 \mathrm{~g}$, respectively, with significant difference $(\mathrm{a}=0.05)$. The mean survivals for each of the treatments were $75.94 \%, 73.96 \%, 74.53 \%$ and $72.25 \%$ which showed no significant difference $(\mathrm{a}=0.05)$ and the average feed conversion factor $(\mathrm{FCA})$ was 2.47 , $2.57,2.69$, and 2.92 respectively. Regarding the physical, chemical and biological water parameters, they were within the recommendations for the culture. We used the experimental design of repeated measurements through Analysis of Variance and Tukey Test $(\mathrm{a}=0.05)$ and concluded that the density with which the best results were obtained was with treatment (T-2) of 120 unit.m ${ }^{-2}$.
\end{abstract}

Keywords: Growth performance; culture densities; greenhouse; Litopenaeus vannamei.

1. Departamento de Acuicultura e Industrias Pesqueras, Facultad de Pesquería, Universidad Nacional Agraria La Molina, Lima (Perú). Email: fgalecio@lamolina.edu.pe

2. Bach. Ing. Pesquera. Facultad de Pesquería. Universidad Nacional Agraria La Molina, Lima (Perú). 


\section{Introducción}

En el Perú, la crianza de langostinos se desarrolla en la costa norte del país, siendo el departamento de Tumbes uno de los que reúne condiciones ambientales apropiadas para este tipo de acuicultura.

Desde sus inicios de la década de los 70 hasta 1999, se ha desarrollado sostenidamente en términos sociales y económicos; durante este tiempo los cultivos de langostinos han enfrentado graves problemas por la presencia de diversas enfermedades, la mayoría de las cuales se han superado con mejoras tecnológicas y manejo genético, a excepción del virus de la mancha blanca (WSSV) de origen asiático, que a fines de agosto de 1999 llega al Perú produciendo devastadores efectos.

Es así que un número mayoritario de granjas fueron convertidas de semi-intensivos a intensivos con una tendencia de disminuir significativamente el recambio de agua (hasta el punto de recircular el agua dentro de la granja) debido a la aparición del virus de la mancha blanca en América Latina (Jory, 2001). Además, estudios realizados durante el año 2001 en el Centro Nacional de Acuicultura e Investigaciones Marinas (CENAIM), en la estación experimental PESGLASA-Ecuador, demostraron que con temperaturas de agua de $33{ }^{\circ} \mathrm{C}$ el virus de la Mancha Blanca no provoca la muerte del langostino Litopenaeus vannamei (CENAIM, 2002).

El uso de invernaderos se convirtió en una alternativa alentadora para la industria de crianza de langostinos, porque permitía reactivar las áreas de cultivo que fueron abandonadas después de la aparición del WSSV, en los últimos años, y como una opción factible para desarrollar un cultivo con bajo riesgo de mortalidad e infección, de crecimiento rápido, mejores tasas de sobrevivencia, mayores rendimientos (hasta cuatro ciclos por año) y una reducción de agua de recambio en los estanques, como estrategia para prevenir el ingreso de vectores de transmisión de enfermedades (Suárez, 2003).

Desde 2008 a la fecha, debido especialmente a los altos costos operativos y a los bajos precios del mercado, el uso de invernaderos se redujo de 100 ha a 40 ha, determinando que los productores que tenían, paralelamente, cultivos semi-intensivos aprendieran a convivir con el virus. Hasta el momento, hay eventos de WSSV pero a un nivel bajo y por lo general se da en situaciones de estrés cuando comienza a cambiar el clima (cambios bruscos de temperatura) o zonas con poca disponibilidad de agua para recambios y baja renovación en el estero que genera mayor carga bacteriana y que finalmente estresa al camarón manifestando el virus.

La actividad de crianza de langostinos se encuentra en un proceso de cambios tecnológicos con la finalidad de lograr mayores niveles de producción y de beneficios económicos. La caracterización del sistema de producción en invernadero describe al cultivo intensivo como aquel que utiliza larvas producidas en laboratorio, con una densidad de siembra de 25 a $150\left(\mathrm{PL} / \mathrm{m}^{2}\right)$, un requerimiento de manejo adecuado, una capacidad de producción de 5,000 a 20,000 ( $\mathrm{k} / \mathrm{ha} / \mathrm{año})$, en un área de 0.1 a 5 ha, utilizando alimento artificial, fertilización, aireación y bombeando el agua repetidamente de 30\% a
100\% diario (Jory, 2001). Al incrementarse la densidad de siembra, el langostino cultivado va a requerir una dieta nutricional más completa debido a la reducida disponibilidad de los alimentos naturales (Mc Intosh, 2000).

El objetivo de la presente investigación fue determinar si la densidad de siembra influye en el comportamiento productivo en un sistema semi-cerrado, con bajo recambio de agua y condiciones intensivas de invernadero.

\section{Materiales y métodos}

El trabajo experimental se llevó a cabo en las instalaciones de la empresa de crianza de langostinos Domingo Rodas S.A., ubicada en la margen izquierda del río Tumbes, distrito San Pedro de los Incas, en el sector Playa Hermosa, a $17,50 \mathrm{~km}$ de la ciudad de Tumbes. El área de estudio correspondió a la zona denominada intensiva y fue utilizada para el experimento el estanque I-7 de un invernadero de 1 ha de espejo de agua revestido con geomenbrana.

El periodo de experimentación se realizó durante los meses de marzo a mayo comprendiendo dos etapas:

Etapa Pre - Experimental: 04 semanas. Construcción e instalación de las 16 jaulas experimentales, llenado y fertilización del estanque, instalación de comederos y monitoreo de la calidad del agua.

Etapa Experimental: 10 semanas. Se trabajó con 2240 juveniles de Litopenaeus vannamei de 3,05 gramos de peso promedio, distribuidos al azar en jaulas experimentales con dimensionesde 1 x 1 x 1,7 m., con armazón de PVC cada una y malla de 1/4 “.

Tabla 1 Distribución de tratamientos

\begin{tabular}{|c|c|c|c|}
\hline $\begin{array}{l}\text { Tratamiento } \\
\mathbf{N}^{\mathbf{0}}\end{array}$ & $\begin{array}{l}\text { Densidad } \\
\text { inicial } \\
\text { Unid } / \mathbf{m}^{2}\end{array}$ & Repeticiones & $\begin{array}{l}\text { Jaula } \\
\mathbf{N}^{\mathbf{0}}\end{array}$ \\
\hline \multirow{4}{*}{ T1 } & \multirow{4}{*}{80} & $\mathrm{~T} 11$ & $\mathrm{~J} 2$ \\
\hline & & $\mathrm{T} 12$ & J5 \\
\hline & & T13 & $\mathrm{J} 10$ \\
\hline & & T14 & $\mathrm{J} 13$ \\
\hline \multirow{4}{*}{$\mathbf{T} 2$} & \multirow{4}{*}{120} & $\mathrm{~T} 21$ & $\mathrm{~J} 1$ \\
\hline & & $\mathrm{T} 22$ & $\mathrm{~J} 7$ \\
\hline & & $\mathrm{T} 23$ & J9 \\
\hline & & $\mathrm{T} 24$ & $\mathrm{~J} 4$ \\
\hline \multirow{4}{*}{ T3 } & \multirow{4}{*}{160} & $\mathrm{~T} 31$ & $\mathrm{~J} 3$ \\
\hline & & T32 & J8 \\
\hline & & T33 & $\mathrm{J} 12$ \\
\hline & & $\mathrm{T} 34$ & $\mathrm{~J} 15$ \\
\hline \multirow{4}{*}{ T4 } & \multirow{4}{*}{200} & T41 & $\mathrm{J} 4$ \\
\hline & & T42 & J6 \\
\hline & & T43 & $\mathrm{J} 11$ \\
\hline & & $\mathrm{T} 44$ & J16 \\
\hline
\end{tabular}


La distribución de los tratamientos en las unidades experimentales fue aleatoria. Se evaluaron las densidades de cultivo de 80 unid $/ \mathrm{m}^{2}$ para el tratamiento uno (T-1), 120 unid $/ \mathrm{m}^{2}$ para el tratamiento dos $(\mathrm{T}-2), 160 \mathrm{unid} / \mathrm{m}^{2}$ para el tratamiento tres (T-3) y $200 \mathrm{unid} / \mathrm{m}^{2}$ para el tratamiento cuatro (T-4), con tres replicas cada uno (Tabla 1)

\section{Evaluaciones}

Los parámetros productivos evaluados fueron: crecimiento, sobrevivencia, biomasa, factor de conversión alimenticia. Los controles biométricos se realizaron cada 2 semanas, así como el seguimiento de los parámetros físicos, químicos y biológicos del agua tales como la temperatura, oxígeno disuelto, $\mathrm{pH}$, salinidad, fitoplancton y zooplancton, transparencia, nutrientes y microbiológicos; también se observó el estado sanitario de los langostinos. Se utilizó alimento comercial para camarón de mar.

\section{Alimentación}

Esta se realizó con el alimento balanceado comercial utilizado por la marca Nicovita, camarón de mar, de 40\%, $35 \%$ y $25 \%$ de proteína y de acuerdo a sus requerimientos proteicos (Mc Intosh, 2000); se llevó a cabo en 2 raciones diarias, previamente pesadas para cada uno de los tratamientos y sus repeticiones, a las 9:00 horas y a las 16:00 horas a través de comederos ubicados en cada jaula experimental.

La ración de alimento a suministrar en cada tratamiento estuvo en función del peso corporal del langostino, según tabla de alimentación recomendada por la empresa para estanques intensivos. La tasa de alimentación que se aplicó al inicio fue del 6\% de biomasa.

\section{Recambio de Agua}

El recambio de agua en estanques es una práctica importante de manejo en el mantenimiento del agua de calidad adecuada. En la crianza de langostinos se aplicó el sistema de cultivo intensivo semi-cerrado con bajo recambio de agua, según requerimiento del estanque. El agua de recambio provenía del reservorio a través del canal de distribución de agua y la renovación de esta se realizaba de 2 a 3 veces por semana. Además, durante el periodo de cultivo se realizaron ingresos de agua para recuperar las pérdidas que ocurrían por filtración, evaporación, sifonado o según requerimiento del estanque.

\section{Parámetros Productivos}

\section{a. Crecimiento}

El control del crecimiento se realizó cada dos semanas empleando el método volumétrico, utilizando para ello una probeta de vidrio. La extracción de la muestra se realizó por la técnica de muestreo aleatorio simple, empleando un chinguillo de $50 \mathrm{~cm}$ de diámetro. El tamaño de la muestra para una población finita fue definida por la fórmula de Cochran, donde se tomó muestras de 22 animales por jaula.

- Para el cálculo del peso promedio, se utilizó la siguiente fórmula descrita por Saldarriaga (2003):
Donde:

$\mathrm{W}_{\mathrm{x}}=$ Peso promedio $(\mathrm{g}), \mathrm{S}$ (wi $\mathrm{x}$ fi) $=$ Sumatoria de pesos observados por su respectiva frecuencia, $n=$ Número de individuos extraídos.

Este peso promedio obtenido es corregido al

$$
W x=\left(\sum(\text { wi } \times \text { fi })\right) / \mathrm{n}
$$

multiplicarse por el factor 1,03 que es el valor del peso específico del agua de mar.

- Para el cálculo del tamaño de muestra se utilizó la fórmula descrita por Cochran (1977):

Donde:

$\mathrm{n}=$ Tamaño de muestra, $\mathrm{Z}=1.96$ (95\% de confianza), $\mathrm{N}=$ Total de la población, $\sigma=$ Desviación estándar, $\delta=$ Precisión o nivel de error aceptado (5\%).

\section{b. Sobrevivencia}

$n=\left(Z^{\wedge} 2 \times N^{\wedge} 2 \times \sigma^{\wedge} 2\right) /\left((N-1) \delta^{\wedge} 2+Z^{\wedge} 2 \delta^{\wedge} 2\right)$

La sobrevivencia real se determinó al final del experimento, cada jaula experimental se desplazó hacia la entrada del invernadero para facilitar la captura de los juveniles. Estos ejemplares fueron atrapados con un chinguillo y colocados en los baldes plásticos para su posterior biometría y conteo. La sobrevivencia fue calculada al final del experimento:

$$
S(\%)=\mathrm{n} / \mathrm{N} \times 100
$$

Donde:

$\mathrm{S}(\%)=$ Porcentaje de sobrevivencia, $\mathrm{n}=$ Número de individuos contados, $\mathrm{N}=$ Número de individuos sembrados.

\section{c. Incremento de Peso}

El incremento de peso se determinó mediante la fórmula de Hopkins (1992) citada por Gutiérrez (1999):

$$
\Delta W=W f-W i
$$

Donde:

$\Delta \mathrm{W}=$ Incremento de peso $(\mathrm{g}), \mathrm{Wf}=$ Peso final $(\mathrm{g}), \mathrm{Wi}=$ Peso inicial (g)

\section{d.Tasa de crecimiento}

La tasa de crecimiento se determinó mediante la fórmula de Steffens (1987):

$T C=($ Incremento de peso $(\mathrm{g})$ en un periodo $(\mathrm{t})) /($ Numero de dias en el periodo $(\mathrm{t}))$

\section{e. Factor de Conversión Alimenticia}

El Factor de Conversión Alimenticia para cada uno de los tratamientos se determinó mediante el uso de la siguiente ecuación descrita por Cuzon, G., Lawrence, A., Gaxiola, G., Rosas, C., Guillaume, J. (2004) y por Huet (1998):

Donde:

$\mathrm{Qa}=$ Cantidad de alimento suministrado $(\mathrm{g}), \Delta \mathrm{B}=$ Incremento o ganancia de peso de la biomasa $(\mathrm{g})$

\section{f. Biomasa}

La biomasa se estir $F \boldsymbol{C A}=\mathrm{Qa} / \Delta \boldsymbol{B}_{\mathrm{yo}}$, mediante la 
fórmula descrita por Saldarriaga (2003):

Donde:

$\mathrm{B}=$ Biomasa estimada $(\mathrm{k}), W_{y r}=$ Peso promedio $(\mathrm{k})$,

$\mathrm{No}=$ Población inicial, $\mathrm{S}=$ Sobrevivencia $(\%)$

Registro de parámetros físico-auímicos v biológicos. El registro de $B=W_{p r} \times S \times N_{0} / 100$ biológicos de la calidad de agua se determinó de acuerdo a la Tabla 2:

\section{Control Sanitario}

Se observó el aspecto externo de los langostinos con el fin de conocer las condiciones sanitarias y detectar síntomas de alguna enfermedad tal como: deformidades en el rostro, abdomen o apéndices; flexión del musculo abdominal o músculo acalambrado; apéndices con machas rojas y fosforescentes (periópodos, pleópodos y urópodos); flacidez del músculo abdominal; enanismo; opacidad del músculo abdominal; laceraciones; lesiones melanizadas; branquias oscuras o negras.

\section{Diseño experimental y evaluación estadística}

En el análisis estadístico de los datos se evaluaron: el crecimiento, la sobrevivencia y la biomasa del langostino. Se planteó la siguiente hipótesis:

-(Hp): No existe diferencia significativa en el efecto de diferentes densidades de siembra de langostino (Litopenaeus vannamei) sobre el crecimiento, sobrevivencia y biomasa.

-(Ha): Existe diferencia significativa en el efecto

Tabla 2. Evaluaciones físico-químicas y biológicas de diferentes densidades de siembra de langostino (Litopenaeus vannamei) sobre el crecimiento, sobrevivencia y biomasa.

\section{a.Crecimiento}

Se empleó el modelo aditivo lineal, correspondiente al Diseño de Mediciones Repetidas descrita por Montgomery (2002):

En esta ecuación los efectos $\tau_{i}, \beta_{j}$ y $\gamma_{k}$ fueron fijados por el investigador a fin de realizar el estudio, entonces podemos decir que es un modelo de efectos fijos. Este modelo se aplicó debido a que las mediciones se

$$
Y_{i j k}=\mu+\beta_{j}+\tau_{i}+\gamma_{k}\left(\tau_{i}\right)+\varepsilon_{i j k}
$$

realizaron cada dos semanas sobre los mismos individuos durante el experimento. Además, se consideraron todas las variables que pueden intervenir en el crecimiento en peso del langostino.

Los datos de crecimiento del langostino se analizaron tomando como criterio de evaluación el peso promedio. Para esta variable se procedió a realizar el análisis de variancia (ANVA; $\boldsymbol{\alpha}=0,05$ ) con mediciones repetidas para determinar si hay diferencias estadísticas significativas entre los tratamientos. Adicionalmente, se procedió a realizar las comparaciones entre los promedios mediante la prueba de Tukey (Ostle, 1973). Por otra parte, se procedió a evaluar las variables de sobrevivencia y biomasa con el modelo aditivo lineal para el diseño completamente al azar, Reyes (1980):

\section{b.Sobrevivencia}

En el análisis de sobrevivencia, se utilizó el número de

\begin{tabular}{|c|c|c|}
\hline PARÁMETRO & FRECUENCIA & MÉTODO \\
\hline Temperatura de agua $\left({ }^{\circ} \mathrm{C}\right)$ & 3 veces/día & $\begin{array}{l}\text { Lectura directa, en un oxímetro modelo YSI- } \\
57 \text { con termómetro incorporado } \\
\text { rango: }-5 \text { a } 45^{\circ} \mathrm{C}\end{array}$ \\
\hline Oxígeno Disuelto (mg/l) & 3 veces/día & $\begin{array}{l}\text { Lectura directa oxímetro modelo YSI- } 57 \\
\text { Rango: } 0 \text { a } 15 \text { ppm }\end{array}$ \\
\hline Salinidad (g/l) & 1 vez/día & $\begin{array}{l}\text { Refractómetro manual } \\
\text { American Optical }\end{array}$ \\
\hline pH & 2 veces/día & Potenciómetro digital -HANNA \\
\hline $\begin{array}{l}\text { Microbiología del Agua } \\
\text { (U.F.C/ml) }\end{array}$ & 2 veces/semana & $\begin{array}{l}\text { Siembra de muestras de agua en medios } \\
\text { de cultivo TSA, TCBS, Mac Conkey y } \\
\text { Cetrimide }\end{array}$ \\
\hline $\begin{array}{l}\text { Nutrientes(mg/l) } \\
\left(\mathrm{NH}_{3}, \mathrm{NO}_{2}, \mathbf{N}, \mathbf{P}\right)\end{array}$ & 2 veces/semana & Espectrofotómetro modelo DR2000 - HACH \\
\hline Transparencia $(\mathrm{cm})$ & $1 \mathrm{vez} / \mathrm{semana}$ & Lectura directa - Disco Secchi \\
\hline Alcalinidad (ppm) & 2 veces/semana & Probeta graduada \\
\hline $\begin{array}{l}\text { Productividad } \\
\text { (Fitoplancton, zooplancton) } \\
\text { (cel/ml y org/ml) }\end{array}$ & 2 veces/semana & $\begin{array}{l}\text { Sedimentación y lectura en hemocitómetro } \\
\text { NEWBAUER, } \\
\text { Filtración en red zooplanctónica }(100 \text { u) }\end{array}$ \\
\hline
\end{tabular}

Fuente: Elaboración propia. 
langostinos vivos al final del experimento expresado en porcentaje, debido a la naturaleza de la variable se procedió a realizar la prueba de Kruskal - Wallis, el

$$
Y_{i j}=\mu+\tau_{i}+\varepsilon_{i j}
$$

cual es apropiado para el diseño completamente al azar (D.C.A.) para determinar si hay diferencias estadísticas significativas entre los tratamientos.

\section{c. Biomasa}

En el análisis de biomasa se utilizó la cantidad de langostinos expresada en kilos, para esta variable se procedió a realizar un análisis de variancia (ANVA;a $=0,05)$ para un diseño completamente al azar (D.C.A.). Después de analizar los resultados se procedió a realizar la prueba de comparaciones múltiples de Tukey (Ostle, 1973).

Para el análisis estadístico de los datos se utilizó el paquete estadístico R stat y Minitab.

\section{Resultados y discusión}

\section{Crecimiento en peso}

Se reportaron pesos promedios finales de $15,50 \mathrm{~g}, 15,61 \mathrm{~g}$, $14,87 \mathrm{~g}$ y $14,33 \mathrm{~g}$ para los tratamientos a densidades de 80 unid $/ \mathrm{m}^{2}$ (T-1), $120 \mathrm{unid} / \mathrm{m}^{2}$ (T-2), $160 \mathrm{unid} / \mathrm{m}^{2}(\mathrm{~T}-3)$ y 200 unid $/ \mathrm{m}^{2}$ (T-4) (Tabla 3).

Mediante la Prueba de Tukey (Ostle, 1973) se realizaron las comparaciones múltiples entre los pesos promedios de los tratamientos y se observó que no existen diferencias significativas entre los tratamientos de $80 \mathrm{ind} / \mathrm{m}^{2}$ (T-1) y $120 \mathrm{ind} / \mathrm{m}^{2}$ (T-2), pero hay diferencias significativas entre los tratamientos T-1 y T-4 y T-2 y T-4 (Tabla 4).

Los resultados obtenidos en T-1 y T-2 fueron mejores a los reportados por Suárez (2003), porque con una densidad de $115 \mathrm{PL} / \mathrm{m}^{2}$ y con un periodo de cultivo de 83 días, se obtuvo ejemplares de un peso final de $12 \mathrm{~g}$; asimismo, los resultados en T-2 fueron mejores en $0,11 \mathrm{~g}$ a los reportados por Socola, M. (comunicación personal) en el sector Lan Karina en la empresa INY donde también se realizó, de manera comercial, una experiencia con densidad de siembra de $100 \mathrm{ind} / \mathrm{m}^{2}$ y hasta el tiempo que duró la experimentación se obtuvieron ejemplares de $15,50 \mathrm{~g}$ de peso promedio; en cuanto a las sobrevivencias, estuvieron alrededor del 80\%, muy diferente a T-3 (Tabla 4 y Tabla 5).

Con el ANVA se determinó que existen diferencias significativas para las densidades de siembra, los tratamientos y los periodos muestreados. Las densidades

Tabla 3. Resultados Generales: cultivo de Litopenaeus vannamei a diferentes densidades de siembra

\begin{tabular}{|c|c|c|c|c|c|c|c|c|}
\hline \multirow{2}{*}{ Tratamientos } & \multicolumn{2}{|c|}{$80 \mathrm{ind} / \mathrm{m}^{2}$} & \multicolumn{2}{|c|}{$120 \mathrm{ind} / \mathrm{m}^{2}$} & \multicolumn{2}{|c|}{$160 \mathrm{ind} / \mathrm{m}^{2}$} & \multicolumn{2}{|c|}{$200 \mathrm{ind} / \mathrm{m}^{2}$} \\
\hline & Prom. & Desv. St. & Prom. & Desv. St. & Prom. & Desv. St. & Prom. & Desv. St. \\
\hline Peso prom. Inicial (g) & 3.1 & 0.051 & 3.05 & 0.214 & 3.07 & 0.095 & 2.98 & 0.064 \\
\hline Peso prom. Final (g) & 15.5 & 0.783 & 15.61 & 0.915 & 14.87 & 0.531 & 14.33 & 0.383 \\
\hline Sobrevivencia (\%) & 75.94 & 3.733 & 73.96 & 6.064 & 74.53 & 4.093 & 72.25 & 7.263 \\
\hline Biomasa Inicial (g) & 248.2 & 4.099 & 366.3 & 25.744 & 491.2 & 15.179 & 596 & 12.754 \\
\hline Biomasa Final (g) & 941.3 & 55.731 & 1385 & 135.517 & 1772.2 & 90.780 & 2068.7 & 192.483 \\
\hline Alimento Total (g) & 1706.9 & 31.846 & 2579.1 & 10.953 & 3432.1 & 32.586 & 4244.7 & 21.870 \\
\hline $\begin{array}{c}\text { Factor de Conversión } \\
\text { Alimenticia }\end{array}$ & 2.47 & 0.166 & 2.57 & 0.337 & 2.69 & 0.189 & 2.92 & 0.367 \\
\hline
\end{tabular}

Fuente: Elaboración propia.

Tabla 4. Prueba de Tukey $(a=0,05)$ para crecimiento

\begin{tabular}{lccccc}
\hline \multicolumn{2}{c}{ Tratamientos } & $\begin{array}{c}\text { Diferencia de } \\
\text { promedios }\end{array}$ & $\begin{array}{c}\text { Error estándar de } \\
\text { las diferencias }\end{array}$ & $\begin{array}{c}\text { Valor - T } \\
\text { calculado }\end{array}$ & $\begin{array}{c}\text { Valor - P ajustado } \\
\text { (Probabilidad) }\end{array}$ \\
\hline Para T-1 & & & & & \\
& T-2 & $-0,0142$ & 0,1107 & $-0,128$ & 0,9992 \\
& T-3 & $-0,3263$ & 0,1107 & $-2,948$ & 0,0217 \\
Para T-2 & T-4 & $-0,6754$ & 0,1107 & $-6,103$ & 0,0000 \\
& T-3 & $-0,3121$ & 0,1107 & $-2,820$ & 0,0306 \\
& T-4 & $-0,6612$ & 0,1107 & $-5,975$ & 0,0000 \\
Para T-3 & & & & & 0,0121 \\
\hline
\end{tabular}

Fuente: Elaboración propia. 
de siembra experimentadas presentaron diferencias altamente significativas en al menos un par de tratamientos. El valor de $\mathrm{P}$-value es menor al nivel $\mathrm{a}=0,05$, por lo tanto se rechaza la hipótesis nula (Hp) (Tabla 6).

\section{Sobrevivencia}

Las tasas de sobrevivencia obtenidas al final del experimento superan el 70\% para los cuatro tratamientos. Se reportaron sobrevivencias finales de 75,94\%, 73,96\%, $74,53 \%$ y $72,25 \%$ para los tratamientos a densidades de $80 \mathrm{unid} / \mathrm{m} 2$ (T-1),,120 unid/m2(T-2),,160 unid/m2(T-3) y $200 \mathrm{unid} / \mathrm{m} 2$ (T-4) según Tabla 3.

Con la Prueba de Kruskal-Wallis el P-value que se obtuvo fue mayor al $\mathrm{a}=0,05$; por lo tanto no existen diferencias significativas entre los tratamientos y la sobrevivencia no fue afectada por las diferentes densidades de siembra, aceptándose la hipótesis nula (Tabla 7). Como el porcentaje de sobrevivencia promedio es el mismo para los diferentes tratamientos, ya no fue necesario realizar las pruebas de comparaciones múltiples.

\section{Biomasa}

Las biomasas alcanzadas promedio fueron de 941,33g, $1384,99 \mathrm{~g}, 1.772,16 \mathrm{~g}$ y $2.068,71 \mathrm{~g}$; correspondientes a $80 \mathrm{unid} / \mathrm{m}^{2}, 120 \mathrm{unid} / \mathrm{m}^{2}, 160 \mathrm{unid} / \mathrm{m}^{2}$ y $200 \mathrm{unid} / \mathrm{m}^{2}$ respectivamente, que se muestran en la Tabla 3.

Mediante el ANVA para DCA donde el P-value es menor al nivela $=0,05$, se determinó que existen diferencias significativas entre las biomasas ganadas en los diferentes tratamientos, por lo tanto se rechaza la hipótesis nula (Hp) (Tabla 8).

Luego, con la prueba de comparaciones múltiples de Tukey (Ostle, 1973) se observó que existen diferencias significativas entre las diferentes combinaciones de

Tabla 6. Análisis de variancia (ANVA; $\mathrm{a}=0,05)$ para los pesos promedios de Litopenaeus vannamei

\begin{tabular}{lcccccc}
\hline $\begin{array}{c}\text { Fuente de } \\
\text { Variación }\end{array}$ & $\begin{array}{c}\text { Grados de } \\
\text { Libertad }\end{array}$ & $\begin{array}{c}\text { Suma de } \\
\text { Cuadrados }\end{array}$ & $\begin{array}{c}\text { Cuadrados } \\
\text { Medios }\end{array}$ & Fc & P-Value & SIGNIF. \\
\hline Densidad & 3 & 7,316 & 2,439 & 16,59 & 0,000 & S. \\
Tratamiento & 12 & 11,402 & 0,950 & 6,46 & 0,000 & S. \\
Periodo & 5 & 1579,648 & 315,930 & 2149,25 & 0,000 & S. \\
Error & 75 & 11,025 & 0,147 & & & \\
Total & 95 & 1609,391 & & & & \\
\hline
\end{tabular}

$\mathrm{R}-\mathrm{Sq}=99,50 \%$

$\mathrm{R}-\mathrm{Sq}(\operatorname{adj})=99,20 \%$

S. = Existe diferencia significativa.

Fuente: Elaboración propia.

Tabla 7. Prueba de Kruskal-Wallis: Sobrevivencia

\begin{tabular}{ccccc}
\hline Densidad & $\mathbf{N}$ & Mediana & Rango promedio & Valor Z calculado \\
\hline $\mathbf{8 0} \mathbf{i n d} / \mathbf{m}^{2}$ & 4 & 0,7500 & 10,1 & 0,79 \\
$\mathbf{1 2 0} \mathbf{~ i n d} / \mathbf{m}^{2}$ & 4 & 0,7500 & 8,4 & $-0,06$ \\
$\mathbf{1 6 0} \mathbf{~ i n d} / \mathbf{m}^{2}$ & 4 & 0,7500 & 8,3 & $-0,12$ \\
$\mathbf{2 0 0} \mathbf{~ i n d} / \mathbf{m}^{2}$ & 4 & 0,7350 & 7,3 & $-0,61$ \\
Total & 16 & 8,5 & \\
$\mathrm{H}=$ criterio de la prueba & \\
$\mathrm{H}=0,76 \mathrm{GL}=3$ P valor $=0,860$ \\
$\mathrm{H}=0,77 \mathrm{GL}=3$ P valor $=0,857$ (ajuste por empates) \\
Fuente: Elaboración propia.
\end{tabular}

Tabla 8. Análisis de variancia (ANVA; $\mathrm{a}=0,05)$ para las biomasas $(\mathrm{kg})$ de Litopenaeus vannamei

\begin{tabular}{ccccccc}
\hline $\begin{array}{c}\text { Fuente de } \\
\text { Variación }\end{array}$ & $\begin{array}{c}\text { Grado de } \\
\text { Libertad }\end{array}$ & $\begin{array}{c}\text { Suma de } \\
\text { Cuadrados }\end{array}$ & $\begin{array}{c}\text { Cuadrados } \\
\text { Medios }\end{array}$ & Fc & P-Value & SIGNIF. \\
\hline Densidad & 3 & 2,8634 & 0,9545 & 57,19 & 0,000 & S. \\
Error & 12 & 0,2003 & 0,0167 & & & \\
Total & 15 & 3,0637 & & & & \\
\hline
\end{tabular}

$\mathrm{R}-\mathrm{Sq}=93,46 \%$

$\mathrm{R}-\mathrm{Sq}(\operatorname{adj})=91,83 \%$

S.=Significativo

Fuente: Elaboración propia. 
Tabla 9. Prueba de Tukey $(\mathrm{a}=0,05)$ para la Biomasa

\begin{tabular}{ccccc}
\hline Tratamiento & $\begin{array}{c}\text { Diferencia de } \\
\text { promedios }\end{array}$ & $\begin{array}{c}\text { Error estándar de } \\
\text { las diferencias }\end{array}$ & $\begin{array}{c}\text { Valor - T } \\
\text { calculado }\end{array}$ & $\begin{array}{c}\text { Valor - P ajustado } \\
\text { (Probabilidad) }\end{array}$ \\
\hline Para T-1 & & & & \\
T-2 & 0,4437 & 0,09135 & 4,857 & 0,0019 \\
T-3 & 0,8308 & 0,09135 & 9,095 & 0,0000 \\
T-4 & 1,1274 & 0,09135 & 12,341 & 0,0000 \\
Para T-2 & & & & \\
T-3 & 0,3872 & 0,09135 & 4,238 & 0,0055 \\
T-4 & 0,6837 & 0,09135 & 7,485 & 0,0000 \\
Para T-3 & & & & \\
T-4 & 0,2966 & 0,09135 & 3,246 & 0,0309 \\
\hline
\end{tabular}

Fuente: Elaboración propia.

Tabla 10. Resumen de jerarquización de la prueba de Tukey para biomasa

\begin{tabular}{cccc}
\hline TRATAMIENTO & NOMENCLATURA & PROMEDIOS & JERARQUÍA \\
\hline 80 lang $/ \mathrm{m}^{2}$ & $\mathrm{~T}-1$ & 0,9413 & $\mathrm{D}$ \\
$120 \mathrm{lang} / \mathrm{m}^{2}$ & $\mathrm{~T}-2$ & 1,3850 & $\mathrm{C}$ \\
$160 \mathrm{lang} / \mathrm{m}^{2}$ & $\mathrm{~T}-3$ & 1,7722 & $\mathrm{~B}$ \\
$200 \mathrm{lang} / \mathrm{m}^{2}$ & $\mathrm{~T}-4$ & 2,0687 & $\mathrm{~A}$ \\
\hline
\end{tabular}

Fuente: Elaboración propia.

\section{tratamientos (Tabla 9 y Tabla 10).}

En el tratamiento sembrado a una densidad de 80 unid/ $\mathrm{m}^{2}$ se obtuvo un factor de conversión alimenticia (FCA) de 2,47 ; a 120 unid $/ \mathrm{m}^{2}$ se obtuvo un FCA de 2,57; para $160 \mathrm{unid} / \mathrm{m}^{2}$ se obtuvo un FCA de 2,69 y para la densidad de $200 \mathrm{unid} / \mathrm{m}^{2}$ se obtuvo 2,92. Estos resultados demostraron que conforme se incrementa la densidad se desmejora la conversión, sin embargo se está dentro de los rangos obtenidos por las empresas comerciales de crianza de langostinos en Tumbes, bajo la modalidad de invernaderos. La misma empresa Domingo Rodas S.A. reporta que en la campaña 2005-1, en un estanque de 0,77 ha revestido con geomembrana, con una densidad de siembra de $80 \mathrm{ind} / \mathrm{m}^{2}$, obtuvo un FCA de 2,50, en 127 días de cultivo (Altamirano C., comunicación personal). Así mismo, en otros cultivos la fase de engorde realizada en estanques revestidos de geomembranas con cubierta $\left(678 \mathrm{~m}^{2}\right)$ y $\sin$ cubierta $\left(50 \mathrm{~m}^{2}\right)$, con una densidad de siembra de $115 \mathrm{PL} / \mathrm{m}^{2}$, con aireación (28 a $60 \mathrm{HP} / \mathrm{ha}$ ), después de 83 días de cultivo, se obtuvo un FCA de 2,58, una sobrevivencia de 77,4\% y una producción de 10685 $\mathrm{kg} /$ ha para el invernadero, mientras que para el control se obtuvo un FCA de 5,28, una sobrevivencia de 14,3\% y una producción de $1361 \mathrm{~kg} / \mathrm{ha}$ (Suárez, 2003).

Los principales parámetros fisicoquímicos y biológicos de la calidad del agua mostraron que la temperatura promedio de esta, registrada a las 3:00 horas, fue de 32,1 $+/-0,55^{\circ} \mathrm{C}$, a las 15:00 horas fue de $33,2+/-0,50{ }^{\circ} \mathrm{C}$ y a las 20:00 horas fue de $33+/-0,56^{\circ} \mathrm{C}$, encontrándose dentro del valor optimo según Sonnenholzner (2002).

En cuanto al oxígeno disuelto, se establecieron tres tomas diarias para la medición, el promedio general fue de 5,36 +/- 0,19 mg/l con una variación de 4,49+/- 0,18 $\mathrm{mg} / \mathrm{l}$ a las 3:00 horas, $6,84+/-0,49 \mathrm{mg} / \mathrm{l}$ a las $15: 00$ horas y 4,76 +/- 0,14 mg/l a las 20:00 horas, encontrándose cerca y dentro de los límites propuestos por Mc Intosh (2000) donde el nivel óptimo del oxígeno debe ser mayor a $5 \mathrm{mg} / \mathrm{l}$.

Los valores de $\mathrm{pH}$ promedio semanal no variaron, estuvieron dentro del rango permitido $(7,5+/-0,11$ a las 3.00 horas y 7,90 +/- 0,12a las 15:00 horas). Boyd (1996) y LinSunwan (2000) mencionan que el pH no debe ser menor a 7,5 a las $5 \mathrm{~h}$, siendo el rango óptimo de 7,5 a 8,0. La variación no influyó sobre los parámetros evaluados.

Con la salinidad se observó una disminución en la sexta semana, teniendo como valor mínimo de 14,3 ppm. Según Cun (1982) la salinidad no es un factor que limite el crecimiento de los camarones, ya que Litopenaeus vannamei presenta una amplia tolerancia a variaciones de la salinidad, sin embargo la tasa de crecimiento de esta especie disminuye cuando la salinidad excede los $35 \mathrm{ppm}$ o es inferior a $20 \mathrm{ppm}$. La transparencia promedio del agua de cultivo fue de $34,4+/-5,31 \mathrm{~cm}$, dentro del rango aceptable (Boyd, 1996). Para los nutrientes los valores máximos fueron: amonio $\left(\mathrm{NH}_{4}^{+}\right)$de $1,936 \mathrm{mg} / \mathrm{l}$ en la primera semana, nitrito $\left(\mathrm{NO}_{2}^{-}\right)$de $7,011 \mathrm{mg} / 1$ en la décima semana y amoniaco $\left(\mathrm{NH}_{3}^{-}\right)$de $0,329 \mathrm{mg} / \mathrm{l}$ en la segunda semana; en lo que respecta a la alcalinidad se observaron valores mayores a $125 \mathrm{ppm}$, fósforo de 1,090 mg/l. Según Grillo (2002), los nitritos $\left(\mathrm{NO}_{2}^{-}\right)$, se pueden acumular cuando la oxidación de amonio excede a la oxidación de nitrito y son tóxicos a niveles superiores a $2 \mathrm{mg} / \mathrm{l}$, pero los cloruros antagonizan la toma de nitritos y hace 
a los animales más tolerantes, sin embargo en cultivos intensivos concentraciones de 3 a 8 ppm son frecuentes sin causar daños (los cloruros protegen la toxicidad de los nitritos), cuando la alcalinidad total es alta (mayor a 125 ppm). Así mismo, Sonnenholzner (2002) menciona que en los cultivos intensivos bajo condiciones de invernadero, la concentración de amonio en el agua se encuentra por debajo de $0,058 \mathrm{mg} / \mathrm{L}$; por lo tanto podemos considerar que los reportados en el experimento son aceptables.

Para el fitoplancton, el promedio general de diatomeas fue de $56.567+/-24243 \mathrm{cel} / \mathrm{ml}$, de cianofitas fue de $68.233+/-71.419 \mathrm{cel} / \mathrm{ml}$, de clorofitas fue de $2.000+/$ $1.633 \mathrm{y}$ el de dinoflagelados fue de $250+/-500 \mathrm{cel} / \mathrm{ml}$. El zooplancton presentó una concentración promedio de 0,3976 +/- 0,298 org/ml.

En lo que respecta a la productividad del agua, las diatomeas estuvieron en un número reducido de 15.000 $\mathrm{cel} / \mathrm{ml}$ al inicio, incrementándose considerablemente a $89.333 \mathrm{cel} / \mathrm{ml}$ en la segunda semana manteniendo valores altos; las cianofitas, al inicio del cultivo, reportaron valores de $33.000 \mathrm{cel} / \mathrm{ml}$ llegando a su máximo desarrollo hasta $260.667 \mathrm{cel} / \mathrm{ml}$ en la tercera semana, donde hubo presencia de dinoflagelados de $1.000 \mathrm{cel} / \mathrm{ml}$ y predominó la coloración pardo-verdoso en el agua (datos no reportados); las clorofitas, inicialmente, no presentaron concentraciones; solo se pudo observar su presencia a partir de la sexta semana donde se reportó bajas salinidades. Según Mc Intosh (2000) en cuanto al fitoplancton, el nivel óptimo es de $8 \times 10^{3} \mathrm{cel} / \mathrm{ml}$ a $3 \mathrm{x}$ $10^{6} \mathrm{cel} / \mathrm{ml}$.

Al final del cultivo el estado sanitario de los langostinos presento un $69 \%$ con apariencia sana, de aspecto uniforme con brillo normal y liso, cefalotórax y branquias limpias y sin lesiones, transparencia del musculo del abdomen y del cefalotórax, mientras que un $30,5 \%$ presentó manchas rojas y fosforescentes en los apéndices tales como periópodos, pleópodos y urópodos (indicativo de vibriosis), deformidades en rostro, abdomen o apéndices, enanismo, opacidad del músculo abdominal, necrosis bactérica externa, fouling branquial y nado errático.

Según Jory (2001) en condiciones naturales los animales muchas veces resisten bien a los patógenos, pero una vez en condiciones de altas densidades y hacinamiento en las instalaciones de cultivo, el nivel de estrés aumenta y puede desarrollar enfermedad. También puede suceder por cambios súbitos de calidad de agua o aumentos en las tasas de recambio que causen cambios bruscos en la temperatura, salinidad, $\mathrm{pH}$, dureza y otros parámetros.

\section{Conclusiones}

De las evaluaciones de crecimiento, sobrevivencia, biomasa y FCA en condiciones de invernadero, se determinó que la densidad de siembra de $120 \mathrm{ind} / \mathrm{m}^{2}$ (T2) obtuvo los mejores resultados siendo las diferencias altamente significativas.

Las densidades de siembra experimentadas hasta los 70 días de cultivo, presentaron diferencias significativas $(a=0,05)$ sobre el crecimiento expresado en peso promedio, obteniendo los mayores pesos promedios los tratamientos de $80 \mathrm{ind} / \mathrm{m}^{2}$ (T-1) y $120 \mathrm{ind} / \mathrm{m}^{2}$ (T-2), con valores de $15,50 \mathrm{~g}$ y $15,61 \mathrm{~g}$, respectivamente.

\section{Literatura citada}

Boyd, C. E. 1996. Manejo del suelo de calidad de agua en la acuicultura de piscinas. Alabama: Asociación Americana de la Soya, Departament of Fisheries and Aquaculture. Auburn University.

CENAIM. 2002. El CENAIM ante el problema del virus de la Mancha Blanca. Acuicultura del Ecuador, pp. $36-$ 39.

Cochran, W. 1977. Sampling Techniques. John Wiley \& Sons Inc., ( $3^{\circ}$ ed.). New York (USA).

Cun, M. 1982. Guía práctica para la cría de camarón comercial (Penaeus) en Ecuador. Boletín Científico y Técnico, 5 (1), 28. Ecuador: Instituto Nacional de Pesca.

Cuzon, G.; Lawrence, A.; Gaxiola, G.; Rosas, C. y Guillaume J. 2004. Nutrition of Litopenaeus vannamei reared in tanks or in ponds. Aquaculture 235: 513-551.

Grillo, M. 2002. Cultivo intensivo de camarón. Sistema bioseguro y sostenible. Copias de la Conferencia. Tumbes (Perú).

Gutiérrez, F. 1999. Efecto de diferentes niveles de energía digestible y proteina sobre el comportamiento productivo de la Gamitana "Colossoma macropomum". Pisces Characidae. Lima, Perú: Universidad Nacional Agraria La Molina.

Huet, M 1998. Tratado de Piscicultura. ( $4^{\circ}$ edición). Madrid, España: Editorial Mundi Prensa.

Jory, D. 2001. Manejo integral del alimento del camarón, de estanques de producción camaroneros, y principios de bioseguridad. Curso en acuicultura. México: Monterrey Nueva León.

Limsuwan, Ch. 2002. Producción Intensiva de Camarón en áreas con Mancha Blanca (WSSV) - Parte 1. Conferencia 06.04.2002. Tumbes, Perú. Disponible en $<$ http://www. alicorp.com.pe/ohsimages/nicovita/boletines/2002/ bole_0204_01.pdf>

Mc Intosh, R. 2000. Cambiando Paradigmas en el cultivo de langostino: Alimento de baja proteína y estrategias de alimentación. Global Aquaculture Advocate, 3(2): 44-50. Mogollón, M. M. 2003. Efecto de tres densidades de siembra sobre el crecimiento de Penaeus vannamei en jaulas flotantes en mar abierto. (Tesis título profesional de Ingeniero Pesquero). Universidad Nacional de Tumbes, Departamento Académico de Acuicultura. Tumbes, Perú.

Reyes, P. 1980. Bioestadística Aplicada. México: Editorial Trilla.

Saldarriaga, D. E. 2003. Diseño de un sistema de Cultivo Intensivo Semicerrado de Langostino: Langostinera Domingo Rodas S.A. Trabajo de Habilitación. Universidad Nacional de Tumbes, Departamento Académico de Acuicultura. Tumbes, Perú.

Sonnenholzner, S. 2002. Tolerancia de Temperatura. Boletín Informativo, $\mathrm{N}^{\mathrm{o}} 64, \mathrm{pp} .1$

Steffens, W. 1987. Principios fundamentales de alimentación de los peces. Zaragoza, España: Edit. Acribia S.A. 
Enero - Junio 2014

Suárez, S. 2003. Cultivo Intensivo de Langostino Blanco Litopenaeus vannamei (Boone, 1931) en Invernaderos. (Tesis título profesional de Ingeniero Pesquero Acuicultor). Universidad Nacional Federico Villareal. Facultad de Oceanografía, Pesquería y Ciencias Alimentarias. Lima, Perú. 